\title{
The Accuracy of Early Post-Operative Abdominal CT Scan
}

\author{
Michael Shpoliansky ${ }^{1, *}$, Yehonatan Nevo ${ }^{1}$, Yoram Klein ${ }^{2, \#}$, Michal Amitai $^{3, \#}$ \\ ${ }^{1}$ Department of Surgery and Transplantation B, Sheba Medical Center, Tel Hashomer, Ramat-Gan, Israel \\ ${ }^{2}$ Division of Trauma, Sheba Medical Center, Tel Hashomer, Ramat-Gan, Israel \\ ${ }^{3}$ Department of Imaging, Sheba Medical Center, Tel Hashomer, Ramat-Gan, Israel \\ ${ }^{\#}$ These authors contributed equally to this work. \\ *Corresponding author: michael.shpoliansky@sheba.health.gov.il
}

Received January 14, 2019; Revised February 10, 2019; Accepted February 21, 2019

\begin{abstract}
Background: Data is lacking regarding the optimal CT timing for diagnosing intra-abdominal postoperative complications. Our aim was to examine the accuracy of $\mathrm{CT}$ imaging performed in the early post-operative period (POD 1-5) compared to late post-operative period (POD 6-14), in the detection of post-operative complications. Methods: Post-operative CT scans performed in the first 14 days following abdominal surgery, between 2011 and 2013 were reviewed and divided into two groups: early CT scans performed at POD 1-5 and late CT scans performed at POD 6-14. Analysis included clinical and laboratory parameters, radiologic findings and assessment of post-operative course according to $\mathrm{CT}$ scan results. Results: $318 \mathrm{CT}$ scans were performed. The rate of repeated CT scans was higher in the patients included in the early CT scan group compared to the late CT scan group $(23.1 \%$ vs. $11.2 \%, p=0.013)$. There were more re-operations in the early CT group, with no statistical difference $(12.8 \%$ vs. $7.9 \%, \mathrm{p}=0.23)$, whereas drainage procedures were more frequent in the late CT group $(22.1 \%$ vs. $9.0 \%, \mathrm{p}=0.008$ ). Early CT scans were less sensitive in detecting post-operative GI complications ( $56 \%$ vs. $73 \%$, $\mathrm{p}=0.018)$. The accuracy to detect post-operative complications was similar between the groups $(83 \%$ vs. $88 \%$, $\mathrm{p}=0.42$ ). Conclusion: $\mathrm{CT}$ scans performed in the early post-operative period are less sensitive and tend to necessitate repeated scans. The accuracy of abdominal CT examination in detecting post-operative GI complications was not found to be statistically different between the early and late CT scan groups.
\end{abstract}

Keywords: format, microsoft word template, style, insert, template

Cite This Article: Michael Shpoliansky, Yehonatan Nevo, Yoram Klein, and Michal Amitai, "The Accuracy of Early Post-Operative Abdominal CT Scan." Global Journal of Surgery, vol. 7, no. 1 (2019): 19-25. doi: $10.12691 /$ js-7-1-4.

\section{Introduction}

Treating the surgical patient with GI post-operative complications is challenging. Post-operative GI complications, e.g. anastomotic leakage, hollow organ injury, abscess formation and infection can carry a high mortality rate $[1,2]$. Correct diagnosis and treatment reduce mortality. However, diagnosing these complications presents a challenge, especially in the early post-operative period where the classical signs of abdominal pain, tenderness, ileus or fever may be regarded as nonspecific [3].

Computed Tomography (CT) scan is the imaging modality of choice to diagnose intra-abdominal post-operative complications. However, data is lacking on the actual value of postoperative CT scan, as its features may overlap between patients with and without clinically significant complications [3,4]. Abnormal CT findings such as abdominal gas, fat infiltration, bowel wall thickening or free fluid may be interpreted as expected in the early post-operative period $[5,6]$. A localized fluid collection or abscess that is the only manifestation of an anastomotic leak may not be reliably differentiated from a transient postoperative serum collection by CT alone [7]. On the other hand, small amounts of contrast medium extravasation may not always be seen, with studies demonstrating limited CT scan sensitivity between $12 \%-68 \%$ in diagnosing distal anastomotic leak $[8,9]$.

In another study, the sensitivity and accuracy of abdominal CT scanning in the detection of anastomotic leakage was evaluated with the aim to analyze the clinical consequences of false-negative imaging. The low sensitivity and accuracy ( $59 \%$ and $73 \%$ respectively) suggested that a negative CT scan does not rule out anastomotic leakage [10].

Moreover, imaging studies are not consistent in their ability to differentiate between complications that can be managed conservatively, such as contained leaks or simple fluid collections, from complications that require urgent intervention. Even the mere definition of a complication, e.g. an anastomotic leak, varies between studies, as depicted in the systematic review by Bruce et al. [11]. As a result, CT scan may be less valuable in the early post-operative period. 
The absence of clearly pathological CT findings may contribute to less aggressive treatment approach and possibly delay necessary surgical intervention in patients in whom the clinical presentation is subtle [12]. On the other hand, a patient with a clinical scenario that clearly points to a development of a serious complication e.g. anastomotic leak can sometimes be re-operated without a repeated imaging.

The purpose of this study is to describe the performance and to examine the accuracy of abdominal CT imaging performed in early post-operative period between post-operative days (POD) 1-5, in the detection of post-operative complications, as compared to late post-operative period (POD 6-14). The importance of this study is to add data on the implications and consequences of early imaging. That may improve patient management and treatment, and spare un-conclusive tests.

\section{Methods}

This is a retrospective study which included adult male and female patients, who underwent an abdominal surgery at a tertiary hospital over a 3-year period between 2011-2013, and subsequently underwent a post-operative CT examination within 14 days of their primary surgery, as a diagnosing tool for post-operative complications such as leak, perforation and infection. Complications which are not infectious in origin or do not cause infection, e.g. bleeding, ileus or bowel obstruction may have distinct clinical scenarios and may often be detected with other imaging modalities $[7,13]$, and were hence not evaluated in this study.

A total of $430 \mathrm{CT}$ examinations were performed. 112 cases were excluded as follows: 34 CT scans were performed following surgeries that were not considered suitable for the study. 51 scans were performed for a clinical question other than leakage/infectious etiology, e.g. bleeding, bowel obstruction, follow up, and other. 12 scans were inappropriately featured in the electronic file, having technical difficulties with the CT scan itself or performing the CT outside the post-operative 14-day time frame of the study. Additional 15 studies were dismissed from this registry due to unclear clinical course.

The final registry consisted of $318 \mathrm{CT}$ studies which were divided into two groups: The early CT scan group included 78 CT examinations performed at POD 1-5. The late CT group consisted of the remaining 240 examinations performed at POD 6-14.

Data analysis included comparison between early and late CT examinations regarding gender, age, etiology and type of surgery, clinical and laboratory parameters (pain, fever, heart rate, blood pressure, white blood cell count, etc.), radiologic findings, and post-operative course with review of the management according to the CT scan results, including re-intervention (re-operation, drainage or repeated CT scanning), the time interval to re-intervention, mortality and length of ICU stay.

To determine the value of a CT scan in diagnosing postoperative complications, the clinical course of each patient was assessed. If the CT findings suggested that the patient can be managed expectantly, and no intervention was attempted, and indeed the course was uneventful until recovery, the CT scan outcome was labeled as "true negative - TN". In case the patient's condition deteriorated, necessitating intervention by means of re-operation or drainage, then the CT outcome was labeled "false negative - FN". If an intervention was deemed necessary based on the CT findings, by means of re-operation or drainage, and the intervention confirmed the suspicion, it was labeled "true positive - TP". In case the intervention did not confirm the suspicion, it was labeled "false positive - FP". Then a comparison between the two study groups was made.

The accuracy of the CT scan was evaluated with the same method, by comparing between early and late post-operative CT scan outcomes and the subsequent interventions that were made according to those CT scans.

\subsection{Statistical Analysis}

To assess the relation between two categorical variables, Chi-Square Test was used, or the Fisher's Exact Test. The relation between a quantitative variable to a categorical variable with two groups was assessed using the T-Test or the Non-parametric Mann-Whitney Test. All of the statistical analyses were double-sided, and a p-value of $5 \%$ or less was considered significant.

\section{Results}

Between 2011 and 2013, a total of 318 post-operative abdominal CT studies were performed as described earlier. There were no demographic differences between the two study groups. The clinical characteristics of the cohort are listed in Table 1. No inter-group difference was seen in terms of the urgency of surgery, with similar rates of urgent versus elective procedures. There was, on the other hand, a significant inter-group difference in the methods of surgeries performed. In the early CT group, 58\% of the surgeries performed were open surgeries, while in the late CT group, open surgeries were more frequent - 73.9\%, ( $p$-value $=0.015)$. We further divided the cases into subgroups, based on the type of surgery. There were no statistically significant differences in the different types of surgery between the two groups.

The clinical and laboratory signs that led to performing post-operative CT are depicted in Table 2. There were no significant changes between the study groups as to the clinical and laboratory parameters that led to the performance of a CT scan. Wound infection, manifested by pus in the wound or in the surgical drain, was more frequent in the late CT group than in the early CT group $(19.9 \%$ vs. $7.4 \%$ respectively, $\mathrm{p}=0.009)$, so as fever (48.5\% vs. $35.8 \%, p=0.046)$. Tachycardia on the other hand, was noted more frequently in the early $\mathrm{CT}$ group (63\% vs. 50.2\%, p=0.046).

Post-operative CT scan findings are demonstrated in Table 3 . The early CT scan had diagnosed leak either by contrast extravasation or by direct evidence of gas leakage in $24.4 \%$ of the scans, a detection rate that was similar to that of late CT scan $(25 \%)$. The rate of fluid collections and abscesses appeared to be higher in the late CT group. This difference was statistically significant for fluid collection rate $(36.7 \%$ vs. $20.5 \%, p=0.005)$. There were 
also more abscess formation in the late $\mathrm{CT}$ group, and although this difference was not rendered statistically significant, it was very close to being so $(22.1 \%$ vs. $14.1 \%$, $\mathrm{p}=0.087$ ). The frequency of gas appearance on the postoperative scan, both minimal and substantial, but not direct evidence of gas leakage, was higher in the early CT group.

The post-operative interventions performed following CT scans are outlined in Table 4. During the period elected for the study, 29 patients $(9.1 \%$ of the study population) were re-operated and 60 patients underwent drainage following their primary operation $(18.9 \%$ of the study operation). Although the rate of re-operations following the post-operative CT was higher in the early CT group than in the late CT group $(12.8 \%$ vs. $7.9 \%)$, this difference was not statistically significant $(p=0.23)$. On the other hand, there were significantly more drainage procedures performed in the late CT group (22.1\% vs. 9.0\%, $\mathrm{p}=0.008),[\mathrm{RR} 2.21$ (95\% CI 1.05-4.64)]. The rate of repeated post-operative $\mathrm{CT}$ scans, was significantly higher in the early CT group (23.1\% vs. $11.2 \%, \mathrm{p}=0.013)$, [RR 2.05 (95\% CI 1.2-3.52), reflecting an increase of $106 \%$; absolute risk (AR) increase of $11.9 \%$ ].

The inter-groups comparison in terms of post-operative course is shown in Table 5. The rate of fatal outcome was higher in the early CT group $(\mathrm{p}=0.028)$. The median time leg from the CT scan to post-operative intervention (whether re-operation or drainage), was 2 days in the early CT scan group, versus only 1 day in the late CT scan group but this finding was not statistically significant $(p=0.202)$. There was no statistically significant difference in the length of ICU stay between the groups.

Table 1. Clinical Characteristics

\begin{tabular}{|c|c|c|c|c|}
\hline Characteristic & Early CT \% (N) & Late $\mathrm{CT} \%(\mathrm{~N})$ & Overall \% $(\mathrm{N})$ & $P$ value \\
\hline Urgency of Surgery & & & & 0.35 \\
\hline Elective & $22.5(43)$ & $77.5(148)$ & $60(191)$ & \\
\hline Urgent & $27.6(35)$ & $72.4(92)$ & 40 (127) & \\
\hline Method of Surgery & & & & 0.015 \\
\hline Open & $59.0(46)$ & $74.2(178)$ & $70.4(224)$ & \\
\hline Laparoscopy & $41.0(32)$ & $25.8(62)$ & $29.6(94)$ & \\
\hline Type of Surgery & $(78)$ & $(240)$ & $(318)$ & 0.7 \\
\hline Stomach & $9.0(7)$ & $8.3(20)$ & $8.5(27)$ & \\
\hline Small bowel & $16.7(13)$ & $20.0(48)$ & $19.2(61)$ & \\
\hline Right colon & $11.5(9)$ & $13.3(32)$ & $12.9(41)$ & \\
\hline Left/sigmoid colon & $10.3(8)$ & $7.9(19)$ & $8.5(27)$ & \\
\hline Total/subtotal colectomy & $3.8(3)$ & $3.7(9)$ & $3.8(12)$ & \\
\hline Rectal resection & $5.1(4)$ & $10.4(25)$ & $9.1(29)$ & \\
\hline Closure of colostomy & $0.0(0)$ & $1.2(3)$ & $0.9(3)$ & \\
\hline Appendectomy & $9.0(7)$ & $3.7(9)$ & $5.0(16)$ & \\
\hline POVH & $0.01(1)$ & $1.2(3)$ & $1.26(4)$ & \\
\hline Whipple & $2.6(2)$ & $1.7(4)$ & $1.9(6)$ & \\
\hline Combined & $6.4(5)$ & $7.1(17)$ & $6.9(22)$ & \\
\hline Other & $23.1(18)$ & $21.7(52)$ & $22.3(71)$ & \\
\hline
\end{tabular}

*"Other" category included - explorative laparotomy, non ventral hernia repair, adhesiolysis, take down anastomosis, abdominal lymph node resection, hepatectomy, cholecystectomy, splenectomy, adrenalectomy, distal pancreatectomy.

Table 2. Indication for Post-operative CT Scan

\begin{tabular}{|c|c|c|c|c|}
\hline Characteristic & Early CT \% (N) & Late $\mathrm{CT} \%(\mathrm{~N})$ & Overall \% (N) & $P$ value \\
\hline Pain & $41.0(32)$ & $40.0(96)$ & $40.2(128)$ & 0.91 \\
\hline Fever & $35.9(28)$ & $48.7(117)$ & $45.6(145)$ & 0.046 \\
\hline Tachycardia & $64.1(50)$ & $50.4(121)$ & $53.8(171)$ & 0.046 \\
\hline Hypotension & $12.8(10)$ & $10.4(25)$ & $11.0(35)$ & 0.62 \\
\hline Leukocytosis/Leucopenia & $44.9(35)$ & $56.2(135)$ & $53.5(170)$ & 0.11 \\
\hline CRP & $29.5(23)$ & $31.2(75)$ & $30.8(98)$ & 0.65 \\
\hline Bilirubin & $9.0(7)$ & $8.3(20)$ & $8.5(27)$ & 0.92 \\
\hline Amylase & $3.8(3)$ & $2.9(7)$ & $3.1(10)$ & 0.72 \\
\hline Creatinine & $12.8(10)$ & $8.3(20)$ & $9.4(30)$ & 0.28 \\
\hline Imaging & $11.5(9)$ & $15.0(36)$ & $14.2(45)$ & 0.77 \\
\hline Abdominal Distension & $12.8(10)$ & $16.2(39)$ & $15.4(49)$ & 0.41 \\
\hline Vomiting & $11.5(9)$ & $14.2(34)$ & $13.5(43)$ & 0.69 \\
\hline Pus in Wound/Drain & $7.7(6)$ & $20.0(48)$ & $17.0(54)$ & 0.009 \\
\hline Hemoglobin & $1.3(1)$ & $2.9(7)$ & $2.5(8)$ & 1.0 \\
\hline
\end{tabular}


Table 3. Post-operative CT Scan Findings

\begin{tabular}{|c|c|c|c|c|}
\hline Characteristic & Early CT \% (N) & Late $\mathrm{CT} \%(\mathrm{~N})$ & Overall \% (N) & $P$ value \\
\hline Leak - Contrast Extravasation & $10.3(8)$ & $10.4(25)$ & $10.4(33)$ & 0.89 \\
\hline Leak - Direct Evidence of Gas Leakage & $14.1(11)$ & $14.6(35)$ & $14.5(46)$ & 0.83 \\
\hline Peritoneal Gas - Substantial & $11.5(9)$ & $5.4(13)$ & $6.9(22)$ & 0.015 \\
\hline Peritoneal Gas - Minimal & $30.8(24)$ & $10.0(24)$ & $15.1(48)$ & $<0.001$ \\
\hline Free Peritoneal Fluid & $23.1(18)$ & $26.7(64)$ & $25.8(82)$ & 0.58 \\
\hline Peritoneal Fluid - Minimal & $28.2(22)$ & $32.5(78)$ & $31.4(100)$ & 0.38 \\
\hline Loop Distension & $21.8(17)$ & $23.3(56)$ & $22.9(73)$ & 0.68 \\
\hline Bowel Wall Thickening & $19.2(15)$ & $25.0(60)$ & $23.6(75)$ & 0.24 \\
\hline Fat Infiltration & $42.3(33)$ & $37.1(89)$ & $38.4(122)$ & 0.42 \\
\hline Abscess & $14.1(11)$ & $22.1(53)$ & $20.1(64)$ & 0.087 \\
\hline Fluid Collection & $20.5(16)$ & $36.7(88)$ & $32.7(104)$ & 0.005 \\
\hline Other & $23.1(18)$ & $27.9(67)$ & $26.7(85)$ & 0.45 \\
\hline
\end{tabular}

"Other" category included - Hematoma, pleural changes, gallbladder changes, pneumobilia, tumor necrosis, pneumatosis, thrombi, skin infection.

Table 4. Post-operative Interventions

\begin{tabular}{|l|c|c|c|c|}
\hline Characteristic & Early CT \% (N) & Late CT \% (N) & Overall \% (N) & P value \\
\hline Re-operation & $12.8(10)$ & $7.9(19)$ & $9.1(29)$ & 0.23 \\
\hline Drainage & $9.0(7)$ & $22.1(53)$ & $18.9(60)$ & 0.008 \\
\hline Repeated CT Scan & $23.1(18)$ & $11.2(27)$ & $14.1(45)$ & 0.013 \\
\hline
\end{tabular}

Table 5. Post-operative course

\begin{tabular}{|l|c|c|c|c|c|c|c|}
\hline Characteristic & \multicolumn{2}{|c|}{ Early CT (N) } & \multicolumn{2}{c|}{ Late CT (N) } & \multicolumn{2}{c|}{ Overall (N) } & P value \\
\hline Death & \multicolumn{2}{|c|}{$14.1(11)$} & \multicolumn{2}{|c|}{$5.83(14)$} & \multicolumn{2}{|c|}{$7.86 \%(25)$} & 0.028 \\
\hline & Mean & Median & Mean & Median & Mean & Median & \\
\hline $\begin{array}{l}\text { Time Lag to Post-operative } \\
\text { Intervention (days) }\end{array}$ & 7 & 2 & 4.95 & 1 & 5.39 & 1 & 0.202 \\
\hline ICU days & 1.65 & 0 & 0.85 & 0 & 1.07 & 0 & 0.11 \\
\hline
\end{tabular}

Table 6. CT Scan Outcome Evaluated by Post-operative Course

\begin{tabular}{lcccc}
\hline Characteristic & Early CT \% (N) & Late CT \% (N) & Overall \% (N) & P value \\
\hline True Negative - TN & $64.1(51)$ & $60.4(147)$ & $61.3(198)$ & \\
True Positive - TP & $17.9(14)$ & $27.1(65)$ & $24.8(79)$ & \\
False Negative - FN & $14.1(11)$ & $9.6(23)$ & $10.7(34)$ & \\
False Positive - FP & $2.6(2)$ & $2.1(5)$ & $2.2(7)$ & 0.28 \\
Total & $100(78)$ & $100(240)$ & $100(318)$ & \\
\hline
\end{tabular}

Table 7. Sensitivity, specificity, positive and negative predictive value and accuracy

\begin{tabular}{lcccc}
\hline Characteristic & Early CT \% & Late CT \% & Overall \% & P value \\
\hline Sensitivity & 56 & 73 & 70 & 0.018 \\
Specificity & 96 & 96 & 96 & 1 \\
PPV & 88 & 93 & 92 & 0.33 \\
NPV & 82 & 86 & 85 & 0.56 \\
Accuracy & 83 & 88 & 86 & 0.42 \\
\hline
\end{tabular}

\subsection{CT Scans Outcome Evaluated by Post-operative Course}

In accordance with the methodology described in methods, Table 6 shows that although the rate of FN cases was higher in the early CT group, while the rate of TP cases was higher in the late $\mathrm{CT}$ group, no statistically significant inter-group difference was found $(\mathrm{p}=0.28)$.
Next, sensitivity, specificity, positive predictive value, negative predictive value and accuracy were assessed (Table 7). There was a significantly lower sensitivity of the early CT scan in diagnosing serious post-operative complications necessitating intervention.

Further, the accuracy of post-operative CT outcomes was assessed and compared between the study groups. The accuracy rate was slightly higher in the late CT group, but the difference was not statistically significant. 
Table 8. Post-operative Re-Interventions After False Negative Imaging

\begin{tabular}{ccccc}
\hline Characteristic & Early CT \% (N) & Late CT \% (N) & Overall \% (N) & P value \\
\hline Re-operation & $3.85(3)$ & $3.75(9)$ & $3.77(12)$ & 0.38 \\
Drainage & $10.3(8)$ & $5.0(12)$ & $6.3(20)$ & 0.013 \\
\hline
\end{tabular}

\subsection{Post-operative Re-Interventions after False Negative Imaging}

Table 8 describes the necessary re-interventions following initial FN imaging, which were made accordingly in course of treatment. Among these FN cases, there were significantly more drainage procedures made in the early CT group ( $\mathrm{p}=0.013)$, [RR 2.05 (95\% CI 0.87-4.83), AR increase of $5 \%$ ]. No significant difference was noted in the rate of re-operation following $\mathrm{FN}$ imaging.

\section{Discussion}

$\mathrm{CT}$ scan is the imaging procedure of choice to diagnose intra-abdominal post-operative complications. However, there is insufficient data about the value of CT scanning in the early post-operative CT period, when abnormal CT findings such as abdominal gas, fat infiltration or free fluid may be regarded as expected, while its sensitivity is limited and can potentially lead to a misdiagnosing of a serious condition and to delaying treatment.

This retrospective study was conducted to define the value and to establish the accuracy of abdominal CT imaging performed in the early post-operative period between POD 1-5 in the detection of post-operative complications. It was also compared to late post-operative period (POD 6-14). We aimed to examine the implications and consequences of an early post-operative CT scan compared to the late one.

Minimal amounts of free gas were found in $30.8 \%$ of early CT scans, and more substantial gas amounts on $11.5 \%$, both being significantly more frequent than in the late CT group. Despite that, direct gas leak was only evident in $14.1 \%$ of scans, and that was not different from the gas leakage percentage in the late CT group. These results are similar to the findings of Gayer et al, demonstrating free gas on $44 \%$ of early post-operative CT scans and on $30 \%$ of late CT scans [5]. Similar findings were presented by Power et al showing that free abdominal air may well persist in the second post-operative week [3].

We have found a low CT scan sensitivity of only $56 \%$ in diagnosing clinically significant early post-operative complications (POD 1-5), a factor that was statistically significant when compared to the later post-operative time period (POD 6-14). This finding was comparable to findings by other authors which show limited CT sensitivity in the early post-operative period.

Moreover, the results show that early CT scans are more likely to necessitate a repeated scan. The rate of repeated CT scans was more than twice higher with the early CT scan group $(23.1 \%$ vs. $11.2 \%, p=0.013)$. Whether because of the slightly higher rate of nonsignificant changes on the CT, e.g. abdominal gas, or due to other reasons, this finding suggests that $\mathrm{CT}$ scans ordered in the early post-operative period are prone to different interpretations and uncertainty.

Apart of the radiation hazards with every additional scan, this uncertainty may affect the clinical course of the patient, by possibly delaying a necessary intervention, as there were more drainage procedures in the early CT group. Despite the low sensitivity, the accuracy of the early CT was not inferior to that of the late CT. This can be explained by the high rate of true-negative CT outcomes.

The differences in the time lag from post-operative CT scan to an active post- operative intervention (whether surgery or drainage), were not statistically significant between the two study groups $(p=0.22)$. However, it is easily seen that the median time leg from CT to intervention in the early CT group, was twice longer than with the late CT group (median 2 days vs. 1 day respectively). This could indicate a trend towards a delay of necessary treatment after early performed CT scans, owing, hypothetically, to uncertain CT interpretation.

The rate of death was higher in the early CT group (14.1\% vs. $5.8 \%, p=0.028)$. A possible explanation is simply because these were the more difficult patients, which is why the CT scan was performed early in the first place. It is worth mentioning that a CT scan performed at a later time was not associated with worse outcome. There was no disadvantage with late CT scans, in terms of mortality, ICU stay, or the need for additional postoperative procedures. That may suggest that there is no disadvantage in postponing the CT scan when possible in appropriate situations, to a later period.

The difference in abscess formation rate between the two study groups, although higher in the late CT group, was not statistically significant. That seemingly supports the work by Antevil et al. [14], which showed no significant difference in the diagnostic yield of CT for abscesses between early and late CT groups. However, it was on the verge of clinical significance $(22.4 \%$ vs. $13.6 \%$, $\mathrm{p}=0.087$ ), a finding worth mentioning. Further, the rate of fluid collections detected, was much higher in the late CT group, a finding which was also statistically significant $(36.5 \%$ vs. $19.8 \%, p=0.005)$. Both findings come to terms with the understanding of the inflammatory process, and the known observation that abscesses usually do not develop until the second week following surgery [15].

The rate of drainage procedures in the late $\mathrm{CT}$ scan group was more than twice higher than in the early CT group $(22.1 \%$ vs. $9.0 \%, \mathrm{p}=0.008)$. Moreover, it is evident that the early CT scan will likely not detect a large proportion of fluid collections and abscesses, and that a great portion of them are clinically significant and are not responsive to antimicrobial treatment alone, from the fact that the rate of drainage procedures following a FN interpretation of the CT scan was twice as high in the early CT. 
Of note, the rate of open vs. late surgery was significantly higher in the early post-operative CT group. Is it a plane chance? Or whether, the higher occurrence of urgent CT scans shortly after the operation among the laparoscopic surgeries, reflect a higher rate of early postoperative complications? Indeed, concerns have risen about increased rate of operative complications in laparoscopic vs. open surgeries. Sammour et al. conducted a meta-analysis comparing intraoperative complication rates of laparoscopic and open colorectal resection [16]. Four thousand and fifty-five patients were analyzed in 10 randomized control trials; There was a higher total intraoperative complication rate [odds ratio (OR) 1.37, $\mathrm{P}=0.010]$ and a higher rate of bowel injury in the laparoscopic group (OR 1.88, $\mathrm{P}=0.020$ ). There was no difference in the rate of intraoperative hemorrhage or solid organ injury. The authors were not able to individually identify the postoperative impact of each intraoperative complication.

Another possible explanation for the higher frequency of laparoscopic approach in the early CT group in this study is, that with laparoscopic surgery, without "feeling the tissue", the surgeon might be less sure of his actions and results during the operation. This uncertainty might lead to further uncertainty with CT scan interpretation, and taking more aggressive approach with every sign of possible complication.

\section{Limitations}

This study has several limitations. One limitation is its retrospective nature. The other is the objective difficulty in the CT interpretation, especially in the early postoperative period as discussed earlier, leading to different radiologic definitions to the same findings and the possible variability between radiologists. All CT scans included in the study were made in the same medical center and interpreted by trained radiologists. The most important limitation of the study is its format, in which clinical decisions that were made based on the CT scan outcome may be individual and differ among caregivers, especially when the clinical scenario and laboratory results may not be the same for different patients. All major clinical decisions in this hospital are made by trained general surgeons as the standard care. A partial way to overcome this limitation may be to add more subgroup analysis, by dividing the cases by type of surgery, type of complication, parallel clinical parameters and more. The downside of this stratification would be loss of the generalizability which characterizes this study.

\section{Conclusion}

Abdominal CT scan performed in the early post-operative period between POD 1- 5 for the detection of infectious abdominal complications, are less sensitive and carry more than a two-fold risk of repeating it, compared with CT scan done between POD 6-14.

The accuracy of abdominal CT examination in detecting post-operative GI complications was not found to be statistically different between the early and late CT scan groups. Therefore, an intelligent use of CT scan in the appropriate timing should be based on clinical scenario. In the suitable situation, when a question of abdominal complication arises, it might be wise to refrain from performing an early CT scan, and make a therapeutic decision according to other clinical parameters, or to delay the scan so that its yield will be higher. That may contribute to a better patient management and treatment, and sparing of un-conclusive and potentially harmful tests.

\section{References}

[1] Bokey EL, Chapuis PH, Fung C, Hughes WJ, Koorey SG, Brewer D, Newland RC: Postoperative morbidity and mortality following resection of the colon and rectum for cancer. Diseases of the colon and rectum 1995, 38(5): 480-486; discussion 486-487.

[2] Kingham TP, Pachter HL: Colonic anastomotic leak: risk factors, diagnosis, and treatment. Journal of the American College of Surgeons 2009, 208(2): 269-278.

[3] Power N, Atri M, Ryan S, Haddad R, Smith A: CT assessment of anastomotic bowel leak. Clinical radiology 2007, 62(1): 37-42.

[4] DuBrow RA, David CL, Curley SA: Anastomotic leaks after low anterior resection for rectal carcinoma: evaluation with CT and barium enema. AJR American journal of roentgenology 1995, 165(3): 567-571.

[5] Gayer G, Jonas T, Apter S, Amitai M, Shabtai M, Hertz M: Postoperative pneumoperitoneum as detected by CT: prevalence, duration, and relevant factors affecting its possible significance. Abdominal imaging 2000, 25(3):301-305.

[6] Zissin R, Osadchy A, Gayer G: Computed tomography findings of early abdominal postoperative complications. Canadian Association of Radiologists journal = Journal l'Association canadienne des radiologistes 2007, 58(3):136-145.

[7] Kim KW, Choi BI, Han JK, Kim TK, Kim AY, Lee HJ, Kim YH, Choi JI, Do KH, Kim HC et al: Postoperative anatomic and pathologic findings at CT following gastrectomy. Radiographics : a review publication of the Radiological Society of North America, Inc 2002, 22(2):323-336.

[8] Nicksa GA, Dring RV, Johnson KH, Sardella WV, Vignati PV, Cohen JL: Anastomotic leaks: what is the best diagnostic imaging study? Diseases of the colon and rectum 2007, 50(2): 197-203.

[9] Kornmann VN, Treskes N, Hoonhout LH, Bollen TL, van Ramshorst B, Boerma D: Systematic review on the value of CT scanning in the diagnosis of anastomotic leakage after colorectal surgery. International journal of colorectal disease 2013, 28(4): 437-445.

[10] Kornmann VN, van Ramshorst B, Smits AB, Bollen TL, Boerma D: Beware of false-negative CT scan for anastomotic leakage after colonic surgery. International journal of colorectal disease 2014, 29(4): 445-451.

[11] Bruce J, Krukowski ZH, Al-Khairy G, Russell EM, Park KG: Systematic review of the definition and measurement of anastomotic leak after gastrointestinal surgery. The British journal of surgery 2001, 88(9): 1157-1168.

[12] Khoury W, Ben-Yehuda A, Ben-Haim M, Klausner JM, Szold O: Abdominal computed tomography for diagnosing postoperative lower gastrointestinal tract leaks. Journal of gastrointestinal surgery: official journal of the Society for Surgery of the Alimentary Tract 2009, 13(8): 1454-1458.

[13] Sandrasegaran K, Maglinte DD: Imaging of small bowel-related complications following major abdominal surgery. European journal of radiology 2005, 53(3): 374-386.

[14] Antevil JL, Egan JC, Woodbury RO, Rivera L, Oreilly EB, Brown $\mathrm{CV}$ : Abdominal computed tomography for postoperative abscess: is it useful during the first week? Journal of gastrointestinal surgery: official journal of the Society for Surgery of the Alimentary Tract 2006, 10(6): 901-905.

[15] Fike FB, Mortellaro VE, Juang D, Sharp SW, Ostlie DJ, St Peter SD: The impact of postoperative abscess formation in perforated appendicitis. The Journal of surgical research 2011, 170(1): 24-26. 
[16] Sammour T, Kahokehr A, Srinivasa S, Bissett IP, Hill AG: Laparoscopic colorectal surgery is associated with a higher intraoperative complication rate than open surgery. Annals of surgery 2011, 253(1):35-43. 